\title{
Antenatal Diagnosis of HIV and Syphilis
}

\section{Geeta Shakya*}

National Public Health Laboratory, Teku, Kathmandu, Nepal

\begin{abstract}
The diagnosis of $90 \%$ of people with HIV is the first target of United Nations UNAIDS 90:90:90 visions for 2020, to end the AIDS epidemic by 2030. Antenatal HIV testing is an important step forward in achieving this and the elimination of mother-to-child transmission (EMTCT) of HIV, which is validated by world health organization. This is possible through universal antenatal screening for HIV and effective intervention. A screening tool, useable as a point-of-care (POC) test is essential for HIV testing in the antenatal clinics. We evaluated the performance of SD Bioline HIV/syphilis Duo rapid test kits in this regard, in Nepal and found it a good screening tool with added opportunity for simultaneous diagnosis of HIV and syphilis. Currently, as it is commercially available at affordable cost, screening of pregnant women for syphilis and HIV has become feasible with the potential to form an integral part of improving maternal and child health.
\end{abstract}

Keywords: Pregnancy; HIV testing; Duo rapid test kits

\section{Review}

Improving HIV testing coverage and uptake is a stride towards meeting all aspects of the United Nations UNAIDS 90:90:90 visions for 2020, to end the AIDS epidemic by 2030 - the first target being diagnosis of $90 \%$ of people with HIV [1]. This is especially relevant in the context of HIV testing during pregnancy, as it relates to two lives and is a key factor in the control of vertical transmission (MTCT) of HIV [2].

Women may become pregnant without knowing that they are HIV positive. For them, HIV testing during pregnancy is an opportunity for knowing their HIV status. This also allows time for intervention to prevent transmission (MTCT) to their offspring. For those who test HIV negative, it is reassuring and allows them to avoid the risk of acquiring the disease.

There is $14-48 \%$ chance of vertical transmission from a HIV positive mother to her child [3]. HIV MTCT can occur antenatally (in utero), intrapartum (during labor and delivery) or postpartum (breast feeding) [4].

Antenatal diagnosis of HIV, especially during the first trimester of pregnancy, has obvious benefits to the mother and her baby [5]. From public health stand point, the test uptake rate is very important. Higher uptake rate is conducive to elimination of mother to child transmission (EMTCT) of HIV. This fact has been borne by the high uptake and low transmission rate (MTCT) in the United Kingdom [6]. Countries with low test uptake rate have lagged behind in elimination of the motherto-child transmission (EMTCT) which is considered a milestone in controlling the spread of HIV. Some countries, headed by Cuba [7], have achieved elimination (EMTCT) and have been validated by the WHO, recently.

For achieving the target of elimination best results would be obtained with universal HIV testing. It has been recommended that all pregnant women be offered HIV screening with appropriate counseling and the testing must be voluntary [8]. The WHO global criteria for elimination of mother-to-child transmission (EMTCT) of HIV and syphilis, 2014 has as requisite $\geq 95 \%$ coverage of HIV testing among pregnant women along with $\geq 90 \%$ of ART coverage of HIV-positive pregnant women for WHO validation [9]. Moreover, provider-initiated routine antenatal HIV testing (i.e., an "opt-out" approach) is the standard of care in the developed countries. This has public health importance.
The availability of effective intervention measures for preventing mother-to-child transmission supports universal antenatal screening. However, there is skepticism regarding compatibility of routine antenatal testing and informed consent $[10,11]$. Despite this, no negative effects were noted on ANC attendance, collection of test results, posttest counseling rates or uptake of PMTCT interventions among HIVinfected women during the implementation of routine HIV testing in Zimbabwe [12]. To improve the test uptake and progress in PMTCT, it appears important to ensure that HIV testing be offered equitably and universally.

The availability of a sensitive, easily available and affordable screening tool for HIV testing in the antenatal clinics has been a practical aspect of problem in the prevention of mother-to-child transmission (PMTCT).

Great progress has occurred in the HIV diagnostic technology since the first commercial introduction of HIV antibody tests in 1985 [13]. Currently, rapid diagnostic tests for HIV are also becoming available at affordable cost. These provide diagnostic results conveniently and immediately while the patient is still at the health facility, screening site, or other health care facility. It can be used as a point-of-care (POC) test with the benefit to allow testing, counseling and referrals to be done in one visit. The availability of such tests has made field-diagnosis of HIV inexpensive and technically feasible in low-resource areas, providing easy accessibility to antenatal HIV testing.

We performed a study to evaluate one of the commercially available test kit the SD Bioline HIV/syphilis Duo rapid test kits in Nepal. The results showed that use of this Duo test kit in antenatal clinics/HTCs would meet the long felt need for a good screening tool for their day to day use with the added opportunity for simultaneous diagnosis of HIV and syphilis [14].

*Corresponding author: Geeta Shakya, Former Director of National Public Health Laboratory, Teku, Kathmandu, Nepal; Tel: 977-1-4240217; 9841240312; E-mail: geeta.nphl@gmail.com

Received May 04, 2017; Accepted June 01, 2017; Published June 07, 2017

Citation: Shakya G (2017) Antenatal Diagnosis of HIV and Syphilis. J AIDS Clin Res 8: 701. doi: 10.4172/2155-6113.1000701

Copyright: (c) 2017 Shakya G. This is an open-access article distributed under the terms of the Creative Commons Attribution License, which permits unrestricted use, distribution, and reproduction in any medium, provided the original author and source are credited. 
Intervention strategy for elimination of HIV and syphilis targets the same population groups. HIV PMTCT and prevention of congenital syphilis have similar priorities. Effective pharmacologic intervention necessitates early diagnosis in both.

Syphilis is also called a silent killer disease, because its symptoms frequently go unsuspected but, when co-infected with HIV, it can be formidable, especially for mothers and children. About 12 million new cases of syphilis are diagnosed each year globally, and nearly 10 percent of all HIV-positive persons are also co-infected with syphilis [15-17]. Such a co-morbid state of syphilis and HIV infection is a serious public health problem in the developing countries including sub-Saharan Africa and the most susceptible groups being women and children. Prevalence rates of syphilis among pregnant women can be as much as 17 percent. Over a million pregnant women are infected with syphilis worldwide [18].

Without intervention, women are in danger of transmitting syphilis on to their infants. Co-infected with HIV, syphilis enhances the transmissibility of HIV from mother-to-child (MTCT). So, pregnant women living with both HIV and syphilis are more likely to transmit HIV vertically to their offspring compared to women positive for HIV only.

Like HIV, syphilis too, is a major cause of morbidity and mortality of women and children in resource-limited countries. Untreated syphilis during pregnancy is associated with several deleterious consequences, like intrauterine fetal demise, low birth weight, prematurity and neonatal mortality. Syphilis, however, is curable disease and both HIV and syphilis in infants and young children are almost entirely preventable by eliminating mother-to-child transmission (EMTCT) of these diseases. Nevertheless, although HIV testing has become more accessible for pregnant women in many developing countries as part of routine antenatal care, yet, syphilis testing has to be offered at separate settings, until recently. Once tested for syphilis, many women never turn to receive their HIV test and fail to know their HIV status. They would be deprived of the interventions they so urgently need and inadvertently may pass syphilis on to their offspring. Integrating new rapid syphilis screening with HIV testing for pregnant women like in the SD Bioline HIV/syphilis Duo rapid test kits can have a significant effect in preventing both transmission of syphilis and HIV from mother to child. Duel rapid diagnostic tests for syphilis and HIV have been found equally effective as the single test in antenatal care services [19] as well as in field study [20]. Currently, as it is commercially available at affordable cost, screening of pregnant women for syphilis and HIV has become feasible, easy and cost-effective bearing the potential to form an integral part of improving maternal and child health in the world over.

\section{References}

1. http://www.unaids.org/en/resources/909090

2. CDC MMWR (2002) Revised recommendations for HIV screening of pregnant women. Infectious Diseases in Clinical Practice: Guidelines Section. IDCP 11: 1-33.

3. John GC, Kreiss J (1996) Mother-to-child transmission of human immunodeficiency virus type 1. Epidemiol Rev 18: 149-157.
4. European Collaborative Study (1992) Risk factors for mother-to-child transmission of HIV-1. Lancet 339: 1007-1012.

5. Canadian Paediatric Society (2008) Testing for HIV infection in pregnancy Paediatr Child Health 13: 221-224.

6. Townsend CL, Cortina-Borja M, Peckham CS, de Ruiter A, Lyall H, et al. (2008) Low rates of mother-to-child transmission of HIV following effective pregnancy interventions in the United Kingdom and Ireland, 2000-2006. AIDS 22: 973-981.

7. WHO (2015) WHO validates elimination of mother-to-child transmission of HIV and syphilis in Cuba. Media centre, Geneva.

8. Bernard MB, Handsfield $\mathrm{HH}$, Margaret AL, Robert SJ, Allan WT, et al. (2006) Revised recommendations for HIV testing of adults, adolescents and pregnant women in health-care settings. MMWR 55: 1-17.

9. World Health Organization (2014) WHO global guidance on criteria and processes for validation: Elimination of mother-to-child transmission of HIV and Syphilis.

10. Bennett RR (2007) Antenatal HIV testing and informed consent: An unworkable marriage? J Med Ethics 33: 446-448.

11. de Zulueta P, Boulton M (2007) Routine antenatal HIV testing: The responses and perceptions of pregnant women and the viability of informed consent: A qualitative study. J Med Ethics 33: 329-336.

12. Chandisarewa W, Stranix-Chibanda L, Chirapa E, et al. (2007) Routine offer of antenatal HIV testing ("opt-out" approach) to prevent mother-to-child transmission of HIV in urban Zimbabwe. Bull World Health Organ 85: 821-900.

13. World Health Organization (2004) Rapid HIV tests: Guidelines for use in HIV testing and counseling services in resource-constrained settings.

14. Shakya G, Singh DR, Ojha HC, et al. (2016) Evaluation of SD Bioline HIV/ syphilis Duo rapid test kits in Nepal. BMC Infect Dis 16: 450.

15. World Health Organization (2011) Global prevalence and incidence of selected curable sexually transmitted infections: Overview and estimates. Geneva.

16. Yeganeh N, Watts HD, Camarca M, Soares G, Joao E, et al. (2015) Syphilis in HIV-infected mothers and infants: Results from the NICHD/HPTN 040 study. Pediatr Infect Dis J 34: e52-e57.

17. Newman L, Rowley J, Vander Hoorn S, Wijesooriya NS, Unemo M, et al. (2015) Global estimates of the prevalence and incidence of four curable sexually transmitted infections in 2012 based on systematic review and global reporting. PLoS ONE 10: e0143304.

18. http://www.who.int/reproductivehealth/topics/rtis/syphilis/pregnancy/en/

19. Gaitan-Duarte HG, Newman L, Laverty M, Habib N, González-Gordon LM, et al. (2016) Comparative effectiveness of single and dual rapid diagnostic tests for syphilis and HIV in antenatal care services in Colombia. Rev Panam Salud Publica 40: 455-461.

20. Bristow CC (2015) Contributions to the improvement and understanding of new diagnostics for HIV infection and Syphilis. UCLA. 